\title{
Colorimetry and efficiency of white LEDs: spectral width dependence
}

\author{
Elaine Taylor ${ }^{1}$, Paul R. Edwards ${ }^{1}$, and Robert W. Martin ${ }^{*}, 1$ \\ ${ }^{1}$ Department of Physics, SUPA, Strathclyde University, Glasgow, G4 0NG, U.K.
}

Received ZZZ, revised ZZZ, accepted ZZZ

Published online ZZZ (Dates will be provided by the publisher.)

Keywords (GaN, Colorimetry, LED, spectral width.)

* Corresponding author: e-mail r.w.martin@strath.ac.uk, Phone: +44 (0)141 5483466, Fax: +44 (0)1415522891

\begin{abstract}
The potential colour rendering capability and efficiency of white LEDs constructed by combination of individual red, green and blue (RGB) LEDs are analysed. The conventional measurement of colour rendering quality, the colour rendering index (CRI), is used as well as a recently proposed colour quality scale (CQS), designed to overcome some of the limitations of CRI when narrowband emitters are being studied. The colour rendering performance is maximised by variation of the peak emission wavelength and relative intensity of the component
\end{abstract}

\begin{abstract}
LEDs, with the constraint that the spectral widths follow those measured in actual devices. The highest CRI achieved is 89.5, corresponding to a CQS value of 79, colour temperature of $3800 \mathrm{~K}$ and a luminous efficacy of radiation of $365 \mathrm{~lm} / \mathrm{W}$. By allowing the spectral width of the green LED to vary the CRI can be raised to 90.9, giving values of 82.5 and $370 \mathrm{~lm} / \mathrm{W}$ for the CQS and LER, respectively. The significance of these values are discussed in terms of optimising the possible performance of RGB LEDs.
\end{abstract}

1 Introduction The human eye is incredibly sensitive to variations in shades of white. This poses a considerable challenge to the development of high efficiency LEDbased lighting. The current leading technology for commercial white LEDs involves a blue-emitting InGaN diode pumping a yellow phosphor. Such devices perform remarkably well with efficacies exceeding $200 \mathrm{~lm} / \mathrm{W}$ and wall-plug efficiencies in the region of $60 \%$. LED lifetimes are extremely long, and are often such that ultra-high drive current and high power operation are possible for useful periods of time. Despite these successes there are some potential limitations. Firstly the conversion of blue to yellow light involves an in-built efficiency loss, related to the Stokes' shift. Secondly the gaps in the spectrum emitted by a "blue-plus-phosphor" LED limits the ability to satisfactorily render the colour of everyday objects. Using the colour rendering index (CRI) as a measure of colour rendering ability, typical values are only $75-80 \%$ of that achieved with traditional incandescent bulbs. Finally angular variations in the colour of the emitted light and changing shades of white due to differential ageing of the phosphor and/or LED have to be taken into account. An alternative approach, that would allow these limitations to be overcome, involves a "multi-LED" approach, where the emission from three or more different colour LEDs is combined in a controlled way. The first step is clearly to combine LEDs emitting the three primary colours (red-green-blue, RGB) although for better colour rendering and colour control further different colours would be added.

However, the performance of blue-plus-phosphor LEDs has improved at pace and they retain a considerable lead over the multi-LED alternatives. As discussed by Haitz and Tao [1] there are major complexity challenges when it comes to the separate control and balancing of the emission from multiple LEDs, each varying in a different way with temperature. In this area the colour sensitivity of the human eye works against the multi-LED approach, with small variations in the shade of white being easily detected and disconcerting. The spectra emitted from the blue LED (and colour conversion phosphor) have proved to be much less sensitive to temperature change than the other colour LEDs [1]. A key component of a multi-LED device would be the green emitter, especially since the eye's sensitivity peaks strongly near $555 \mathrm{~nm}$. Producing commercially viable high-power green LEDs remains a research challenge, referred to as the "green gap", and the lack of green LEDs that can perform as well as their blue and red counterparts is an obstacle facing the multi-LED approach. 
Nevertheless it is instructive to assess the advantages that could result from a multi-LED lamp and also to incorporate possible benefits that could follow a filling in of the green-gap, possibly with extra controllability resulting from the variety of different approaches being employed to this end. This paper addresses these questions and evaluates the improvements in colour rendering made possible by a RGB multi-LED approach. Additional performance improvement resulting from a hypothetical ability to tailor the emission spectrum from the green LED is then considered.

\section{Parameters for LED characterisation}

There are a variety of parameters useful for quantifying the performance of white LEDs for lighting or other applications [2]. The most compelling benefit of these light sources is the saving in energy, which can be measured in terms of the luminous efficacy $(\mathrm{lm} / \mathrm{W})$ although it is important to clarify which power is being measured. Defining the luminous efficacy as the amount of luminous flux (in lumens) produced per Watt of input electrical power, it can be seen that two conversions are important. First there is the conversion of input electrical power to optical output power, which is the power conversion efficiency, and then the conversion from output power to output flux, known as the luminous efficacy of radiation (LER, in $\mathrm{lm} / \mathrm{W}$ ). The LER is determined by the spectral distribution of the source and is independent of the intensity. The conventional measure of colour rendering performance is the Commission Internationale de l'Éclairage (CIE) recommended CRI, which involves a summation of the change in chromaticity points (on a CIE 1964 colour space diagram) between a measured and reference light source over a series of Munsell colour samples. Often eight such samples are used, giving a CRI $R_{a}$ value, although extra channels bring in a number of more saturated colours. The highest CRI value (100) indicates no difference in colour rendering from the chosen reference light source, which is often a black body source such as an incandescent light bulb.

However, the CRI is known to have deficiencies when used with spectra composed of narrow bands, such as multi-LED white lamps. Ohno [2] has demonstrated this using LED spectra optimised for high CRI $R_{a}$ and describes a poor correlation between visual evaluation of colour rendering from white LEDs and CRI. To overcome the limitations of CRI for LEDs a different measure of colour rendering, known as the colour quality scale (CQS) has been proposed by researchers at NIST [3]. CQS employs a more recent colour space (CIE 1976) and calculates the differences in chromaticity points between a reference and test source illuminating fifteen test samples, which are all saturated colours. The CQS also used an updated chromatic adaption transform compared to CRI and takes into account other factors important for perceiving certain colours, such as skin tones. Other advantages of the CQS are that, unlike CRI, negative values do not appear. Values for LER,
CRI and CQS are fully determined by the spectrum of a light source, which enables their dependence on different white LEDs to be followed without need to accurately determine the power output.

Additional important parameters for quantifying the colour of LEDs are the Correlated Colour Temperature (CCT) and $D_{u v}$. The CCT is the temperature of an equivalent Planckian source, found by tracking from the chromaticity point of the light source to the Planckian locus using isothermal lines which lie perpendicular to the locus. $D_{u v}$ provides a measure of how far the chromaticity point of the light source lies from the Planckian locus, measured along these perpendicular lines. A white lamp will ideally have a near-zero $D_{u v}$ and lie very close to the Planckian locus. It is not possible to specify an ideal CCT as different values have become accepted as norms in different continents and in different types of location [2]. For example, CCT values near $5000 \mathrm{~K}$ are dominant in Japan, whilst warm-white is specified at $3000 \mathrm{~K}$ or below for homes in the UK and USA. It should be noted that the CQS includes a CCT factor which penalises a light source for CCT values lying outside a desired region (3500 to $6500 \mathrm{~K}$ ).

\section{Performance of RGB multi-LED lamps}

The performance of RGB multi-LED lamps are now assessed using the above parameters. The LED spectra are produced by combining individual spectra from currently available red, green and blue LEDs. The peak emission energy and relative light intensity are considered as variables, although it is recognised that the "green gap" mentioned above limits the available power in the green. The spectral distribution of an RGB LED composed of spectra measured from three separate LEDs with peak emissions at 464, 570 and $615 \mathrm{~nm}$ (and increased red intensity) is shown in Fig. 1. The individual peaks are approximately Gaussian and it is notable that the spectral widths are not equal, in part because different material systems are employed for the red (InGaAlP) and blue/green (InGaN) devices.

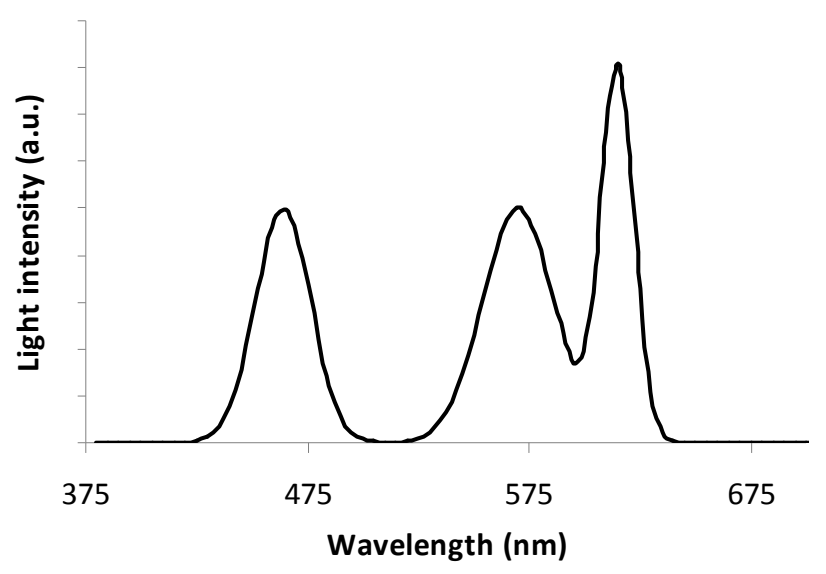

Figure 1 Simulated spectrum of white LED constructed by combining spectra from three separate red, green and blue LEDs. 
An expression for the dependence of the full width at half maxima (FWHM) on peak emission energy is established by measuring the spectra of a number of commercial LEDs and combining with values reported in the literature [4]. Fig. 2 shows example data and two linear regions that will be used to constrain the spectral widths in the multiLED spectral distributions. Further data on FWHM as a function of peak emission energy are reported in Ref. [5], which reports cathodoluminescence (CL) spectra from InGaN/GaN structures. In the region shown in Fig. 2 the CL linewidths agree well with those from the LEDs.

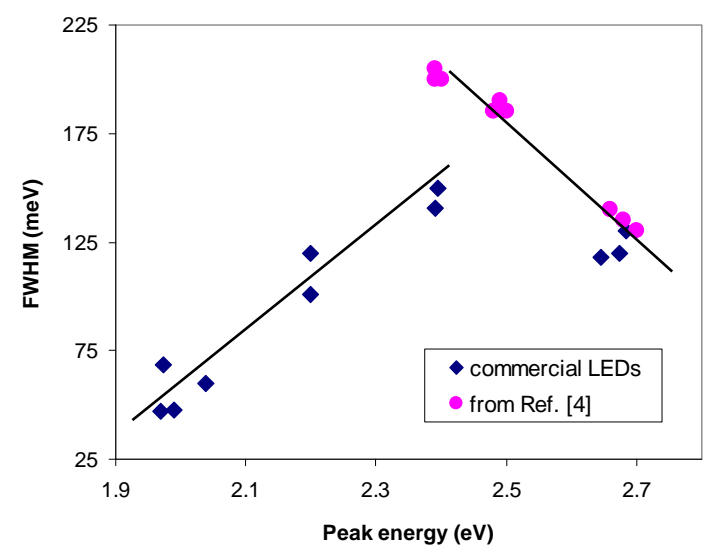

Figure 2 Measured spectral widths of a series of single colour LEDs as a function of their peak emission energy. The straight lines are used to constrain the widths of simulated LED spectra.

Using this constraint the CQS, CRI and LER values of possible RGB LEDs are monitored with a view to maximising the colour rendering performance. The diagrams are plotted using CRI values and comment regarding the equivalent CQS values will be made later. CQS remains a proposed parameter and, despite the recognised shortcomings of CRI for LEDs, the CRI remains the one accepted by the CIE. The approach used was to first separately vary the peak emission wavelength of each component LED, with equal peak intensity for each of the three LEDs, as shown in Fig. 3. The starting point in each case was a (470/540/630 nm) combination and the CRI is seen to be improved by shortening the peak wavelength of the red device, with a CRI of 82 reached for a 470/540/610 nm combination. Using these three peak emission wavelengths the relative intensities were then varied (Fig. 4), looking for further improvements in CRI. Thereafter finer variations in peak emission energies and relative intensities were conducted, in order to iterate to the highest possible CRI. The highest CRI value achieved for these RGB LEDs was 89.5, corresponding to an LER of $365 \mathrm{~lm} / \mathrm{W}$. The CQS value for this LED was 79. An example of this final optimisation step is plotted by line CRI(1) in Fig. 5, which shows the trade-off between CRI and LER. The corresponding CQS values are plotted by the line CQS(1), providing comparison with the CRI values. These CQS values increase with

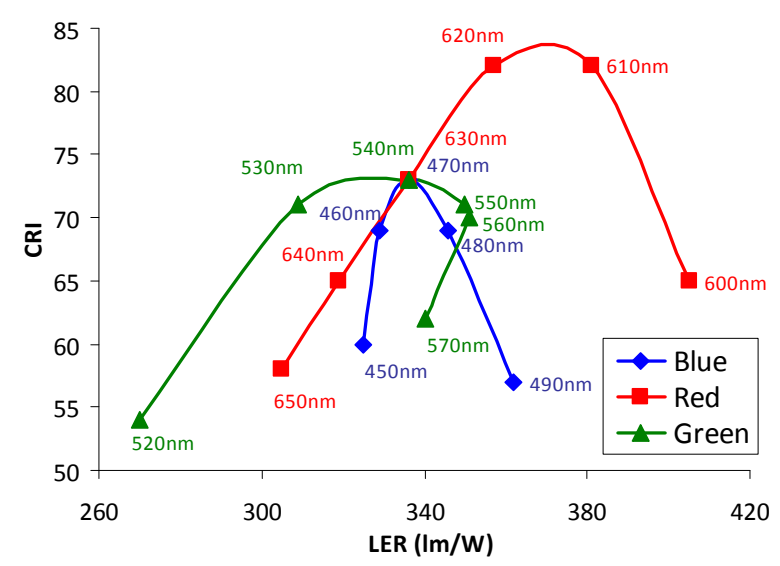

Figure 3 Coarse optimisation of CRI by stepping the peak emission wavelength of each of the three component LEDs, one by one, with constant intensities. Labels show the peak wavelength of the stepped device and identify the full ranges considered.

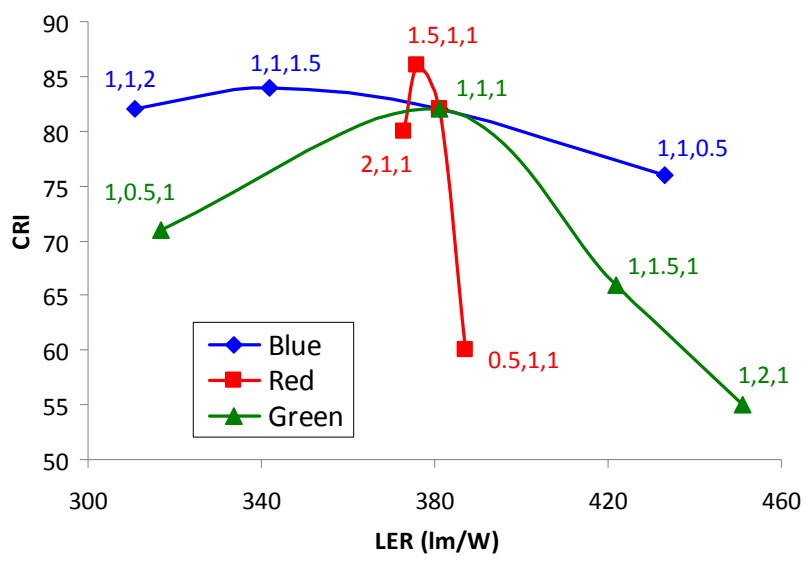

Figure 4 Further optimisation of CRI by adjustment of the relative intensities, one by one, of the 470, 540 and $610 \mathrm{~nm}$ component LEDs. The labels show the relative RGB intensities.

LER along this line but it must be noted that this does not reflect the best CQS values that are achievable for these LERs, which are almost constant at 81. A significant contributor to the different behaviours of CRI and CQS is the non-uniformity of the red region within the old chromaticity diagram used for CRI.

These values could be further increased if the spectral width and relative efficiency of the component LEDs could be freely varied. As an example, by varying the spectral width of the green LED it was possible to reach CRI and CQS values of 90.9 and 82.5, respectively, with the LER reaching $370 \mathrm{~lm} / \mathrm{W}$. The optimum value corresponds to inclusion of a green LED with a peak emission of $547 \mathrm{~nm}$ and a FWHM of $158 \mathrm{meV}$. This spectral width is approximately 1.3 times that normally achieved for an InGaN/GaN LED emitting at this wavelength. In future, such control of FWHM may be achievable using band-gap engineering techniques, such as graded interfaces and/or asymmetric 


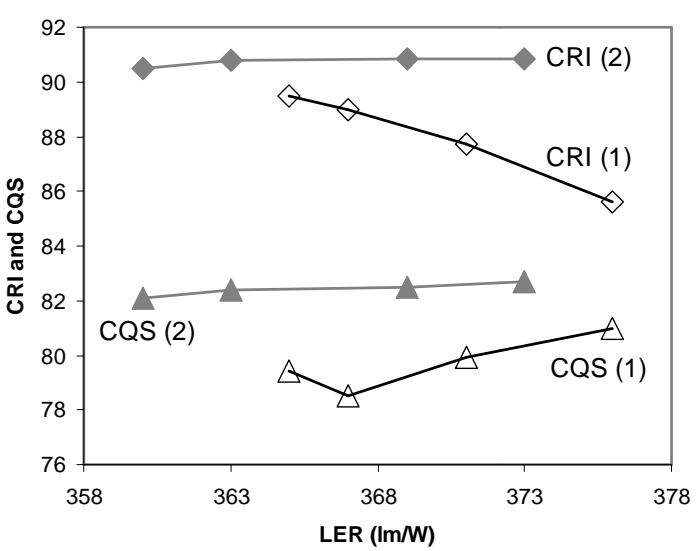

Figure 5 CRI and CQS values for the LEDs showing the highest colour rendering performance. The FWHMs are constrained as in Fig. 2 for the open data-points (lines 1) and unconstrained for the filled points (lines 2). As discussed in the text, these CRI and CQS values are not always the best achievable at constant LER, but show variations in CRI and CQS (and the LER that results) as peak emission energies, intensities and/or linewdiths are varied.

wells. Comparison of the CRI and CQS values is again provided in Fig. 5, using equivalent LER values along the lines labelled (2). In this case the CRI and CQS values both follow the same trend. Fig. 5 shows that the highest CRI and CQS values can be maintained over a range of LER values. In these cases the adjustment of the green spectral width is an important factor to allow increasing efficiency while keeping the colour rendering performance as high as possible. At the same time the CCT values vary within the range $3700-3900 \mathrm{~K}$ and $D_{u v}$ values are in the region of -0.004 . Table 1 provides a comparison of the CRI, CQS, CCT and LER values for typical low-cost commercial white LEDs and an incandescent bulb alongside the optimum values achieved for the RGB LEDs described above. It is noticeable that the CQS exceeds the CRI for the warm white LED, but not for the white LED. This again reflects the improved handling of the red shades by CQS. The improved LER and colour rendering of RGB

Table 1 Comparison of colour rendering and efficiency parameters for various white light sources.

\begin{tabular}{|c|c|c|c|c|}
\hline Light Source & CRI & CQS & CCT (K) & $\operatorname{LER}(\operatorname{lm} / \mathrm{W})$ \\
\hline $\begin{array}{l}\text { Optimum RGB LED, } \\
\text { constrained FWHM }\end{array}$ & 89.5 & 79 & 3810 & 365 \\
\hline Optimum RGB LED (\#1) & 90.9 & 82.5 & 3710 & 370 \\
\hline Optimum RGB LED (\#2) & 90.8 & 82.5 & 3910 & 363 \\
\hline $\begin{array}{l}\text { Commercial white LED } \\
\text { (at } 30 \mathrm{~mA} \text { ) }\end{array}$ & 77 & 75 & 5870 & 319 \\
\hline $\begin{array}{l}\text { Commercial warm white } \\
\text { LED (at } 30 \mathrm{~mA} \text { ) }\end{array}$ & 66 & 70 & 3200 & 347 \\
\hline Fluorescent strip light & 88 & 85 & 3620 & 338 \\
\hline Incandescent bulb & 98 & 96 & 2700 & 137 \\
\hline
\end{tabular}

white LEDs is evident.

The individual CRI values of the various colour channels (Munsell and saturated) are shown in Fig. 6 for the optimised RGB LEDs, with and without the constraint on the green spectral width. R1 to R8 are those used in the calculation of CRI $R_{a}$, whereas the additional channels are factored into the CQS calculations. It can be seen how the increase in the green spectral width has increased a number

(a)

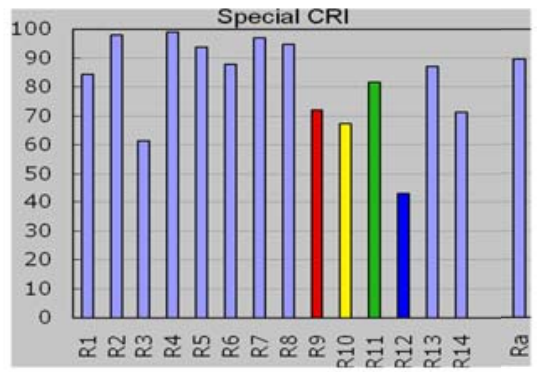

(b)

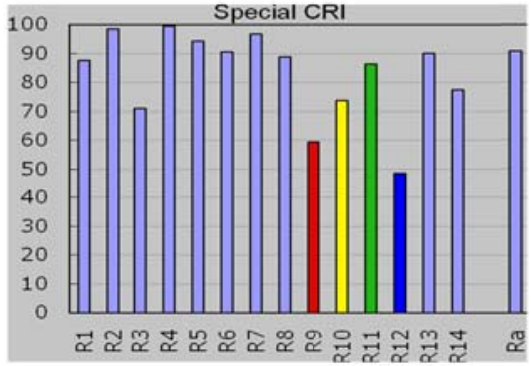

Figure 6 Optimised CRI values of individual colour channels for a 3-LED white lamp using (a) a constrained FWHM for the green LED and (b) an optimised value for the green spectral width.

of the R1-R8 values, most notably R3, R1 and R6. The values have increased for all the saturated colours apart from the red. This results in the greater increase for CQS, compared to CRI, between these two LEDs and the increased green spectral width has produced a light which is more suitable for saturated colours.

\section{Conclusion}

The colour rendering capability of RGB white LEDs has been analysed, including comparison of CRI , CQS and CCT indices. A CRI of 89.5, and CQS of 79, is achieved by variation of the peak emission wavelength and relative intensity of the component LEDs, with the constraint that the spectral widths follow those measured in actual devices. By allowing the spectral width of the green LED to vary the CRI can be raised to 90.9 and the CQS to 82.5. This uses a $547 \mathrm{~nm}$ emitter with a FWHM of $158 \mathrm{~nm}$. The luminous efficacies of radiation are in the region of 370 $\mathrm{lm} / \mathrm{W}$, enhanced over those in other white light sources.

Acknowledgements We are grateful to Wendy Davis (NIST) for helpful advice and the U.K. EPSRC for funding through grants including the Programme Grant "Lighting the Future”, EP/I012591/1. 


\section{References}

[1] R. Haitz and J.Y. Tsao, phys. stat. sol. (a) 208, 17 (2011).

[2] Y. Ohno, Proc. of SPIE Vol. 5530, p88 (2004).

[3] W. Davis and Y. Ohno, Optical Engineering 49, 033602, (2010).

[4] K. P. O’Donnell, T. Breitkopf, H. Kalt, W. Van der Stricht, I. Moerman, P. Demeester, and P. G. Middleton, Appl. Phys. Lett. 70, 1843 (1997).

[5] R.W. Martin, P.R. Edwards, K.P. O’Donnell, E.G. Mackay and I.M. Watson, phys. stat. sol. (a) 192, 117 (2002) 